EPJ Web of Conferences 70, 00016 (2014)

DOI: 10.1051/epjconf/20147000016

(C) Owned by the authors, published by EDP Sciences, 2014

\title{
Measurements of the $Z$ boson in lepton pair decays in heavy ion collisions at ATLAS
}

\author{
Valery Pozdnyakov ${ }^{1, a}$ on behalf of the ATLAS Collaboration \\ ${ }^{1}$ Joint Institute for Nuclear Research, Dubna, Russia
}

\begin{abstract}
Measurement of $\mathrm{Z}$ boson production in $\mathrm{Pb}+\mathrm{Pb}$ collisions at $\sqrt{s_{N N}}=2.76 \mathrm{TeV}$ with the ATLAS detector, obtained for approximately $0.15 \mathrm{nb}^{-1}$ data collected in 2011 $\mathrm{LHC}$ run is presented. The $\mathrm{Z}$ bosons are reconstructed in their di-electron and di-muon decay channels. The results from both channels are found to be consistent and hence combined. Within the statistical and systematic uncertainties, the per-event $\mathrm{Z}$ boson yield integrated over rapidity $\left|y^{Z}\right|<2.5$, is found to be proportional to the number of binary collisions calculated by the Glauber model. The elliptic flow coefficient of the $\mathrm{Z}$ boson azimuthal distribution with respect to the event plane is found to be consistent with zero.
\end{abstract}

\section{Introduction}

The extensive heavy ion (HI) studies [1] performed at the Relativistic Heavy Ion Collider (RHIC) at BNL, and the Large Hadron Collider (LHC) at CERN, have established that the hot and dense matter produced in high energy HI collisions leads to a significant energy loss for energetic color charge carriers penetrating such a medium. In order to understand this so called jet quenching phenomenon, it is important to measure the production rates of these carriers before the energy loss. The best candidates to make such measurements are the particles which do not experience strong interactions. This proceedings presents a measurement of $\mathrm{Z}$ bosons via their electron and muon decay channels in $\mathrm{Pb}+\mathrm{Pb}$ collisions at $\sqrt{s_{N N}}=2.76 \mathrm{TeV}$ [2]. The $\mathrm{Z}$ boson yield is found to be proportional to the average number of binary nucleon-nucleon collisions $\left.\left(<N_{\text {coll }}\right\rangle\right)$ estimated within the framework of the Glauber model [3]. The analysis of the elliptic flow coefficient $\mathrm{v}_{2}$ of the $\mathrm{Z}$ boson angular distribution shows no correlation between the particle emission angle and the azimuthal direction of the event plane, confirming that the $\mathrm{Z}$ boson yield is not affected by the asymmetric initial collision shape.

\section{ATLAS detector}

The ATLAS detector [4] at the LHC almost surrounds the entire solid angle in the collision point. It contains an inner tracking detector located in a thin superconducting solenoid, electromagnetic and hadronic calorimeters, and a muon spectrometer incorporated with three superconducting toroid magnets. The pseudorapidity $(\eta)$ domains covered by different detector systems are shown in Table

\footnotetext{
a e-mail: Valeri.Pozdnyakov@cern.ch
} 


\section{EPJ Web of Conferences}

Table 1. Pseudorapidity coverage of the main ATLAS subsystems.

\begin{tabular}{|c|c|}
\hline detector system & $|\eta|$ \\
\hline Inner Detector (ID) & $<2.5$ \\
Electromagnetic Calorimeters & $<3.2$ \\
Forward Calorimeters (FCal) & $3.1-4.9$ \\
Muon Spectrometer (MS) & $<2.7$ \\
\hline
\end{tabular}

1. The Inner Detector (ID), immersed in a $2 \mathrm{~T}$ axial magnetic field and intended for the charged particle reconstruction, consists of a high-granularity silicon pixel detector, silicon microstrip and a transition radiation trackers. The electromagnetic calorimetry is provided by a barrel and an endcap high-granularity lead liquid-argon (LAr) calorimeters, with an additional thin LAr presampler covering $|\eta|<1.8$. The LAr forward calorimeters provide both electromagnetic and hadronic energy measurements at large pseudorapidity. The muon spectrometer (MS) comprises separate trigger and precision tracking chambers measuring the deflection of muons in the magnetic field generated by superconducting air-core toroids. The precision chamber system includes three layers of monitored drift tubes (MDT), completed with cathode strip chambers (CSC) in the innermost layer of the forward region, where the background is the highest. The muon trigger system covers the range $|\eta|<2.4$ with resistive plate chambers in the barrel, and thin gap chambers in the end-cap regions.

\section{Analysis}

This analysis uses the 2011 LHC lead-lead collision data collected with the ATLAS detector at the LHC, and corresponds to an integrated luminosity of approximately $0.15 \mathrm{nb}^{-1}$. These data were collected by the ATLAS three-level trigger system [5]. The Z bosons are reconstructed in $e^{+} e^{-}$or $\mu^{+} \mu^{-}$ decay channels. The electron candidates were identified at the first trigger level (L1) as an energy cluster formed with $(\eta, \phi)=(0.1,0.1)$ trigger towers of the electromagnetic calorimeter, except the transition region between the barrel and the end-cap calorimeters. The candidates were then required to pass the standard ATLAS electron reconstruction algorithm [6], which requires the matching of a track to an electromagnetic energy cluster. Furthermore, a criteria for the balance between the track momentum, cluster energy and shower shape variables, was also required. The electron candidates are also required to have transverse energy $E_{\mathrm{T}}>20 \mathrm{GeV}$ and with in $|\eta|<2.5$. The selection of the muon candidates was performed using all three levels of the trigger system. The L1 muon trigger searches for patterns of hits consistent with muons of a certain transverse momentum, $p_{\mathrm{T}}$, in the trigger chambers within $|\eta|<2.4$. Then, a full scan muon reconstruction was performed by the high level trigger to identify muons with $p_{\mathrm{T}}>10 \mathrm{GeV}$. Single muons were reconstructed with varying levels of quality. High quality muons were reconstructed in both the MS and ID subsystems, as well as with a good match to the event vertex. It is required that at least one muon in each pair, matched to the trigger, should be of such quality. If both muon candidates have high quality, the $p_{\mathrm{T}}$ threshold is set to $10 \mathrm{GeV}$. If the second muon fails this condition, both muons are required to satisfy $p_{\mathrm{T}}>20 \mathrm{GeV}$. Once identified, electron or muon candidates are then paired with oppositely charged electron or muon. Pairs with an invariant mass between 66 and $102 \mathrm{GeV}$ were accepted as $\mathrm{Z}$ boson candidates. The combinatorial background is estimated to be $5 \%$ for $\mathrm{Z} \rightarrow e^{+} e^{-}$and $1 \%$ for $\mathrm{Z} \rightarrow \mu^{+} \mu^{-}$channels. The invariant mass distributions of the selected events together with estimated 


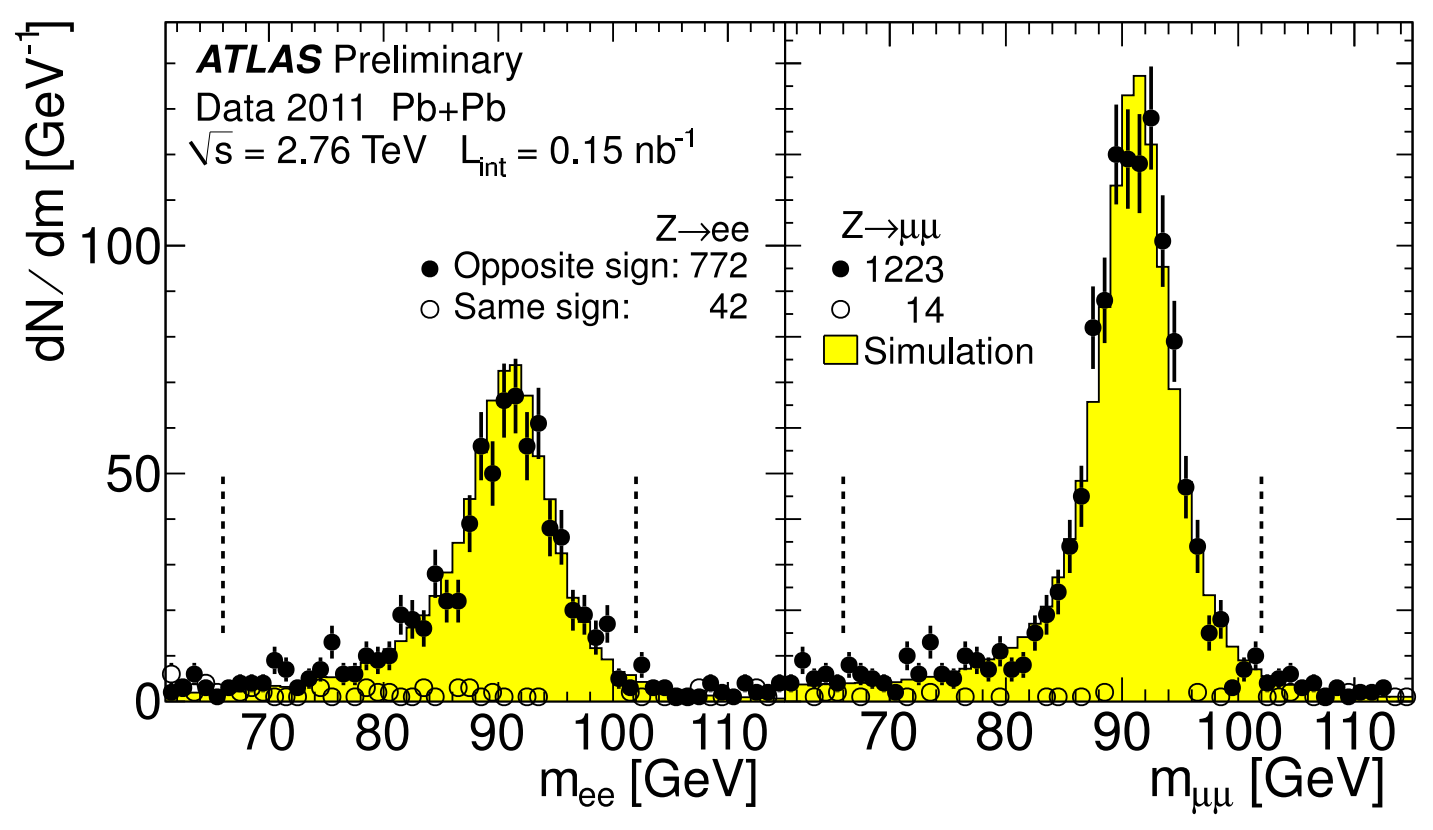

Figure 1. The invariant mass distributions of $\mathrm{Z} \rightarrow e^{+} e^{-}$(left) and $\mathrm{Z} \rightarrow \mu^{+} \mu^{-}$(right) in data (points) and simulation (histogram) reweighted to match the centrality distribution in data and normalized in the region $66<\mathrm{m}_{\mathrm{Z}}<116$ $\mathrm{GeV}[2]$.

combinatorial backgrounds are shown in Fig. 1. The backgrounds from electroweak processes [7] and top pair decays [8] are small compared to the combinatorial background, and their contributions are neglected. The $\mathrm{Z}$ reconstruction efficiency is based on overlay of PYTHIA (version 6.425) [9] Z $\rightarrow l^{+} l^{-}$ events into simulated HIJING (version 1.38b) [10] $\mathrm{Pb}+\mathrm{Pb}$ events, requiring $66<\mathrm{m}_{\mathrm{Z}}<116 \mathrm{GeV}$ and $\left|\mathrm{y}^{\mathrm{Z}}\right|<2.5$. The response of the ATLAS detector to the generated particles was modeled using GEANT4 [11]. The main sources of systematic uncertainties over the measured yields in both lepton channels are associated with the accuracy to which the corrections applied to the data can be calculated. The efficiency and resolution uncertainties depend on the decay kinematics of $Z$ bosons and are on average $8 \%(5.5 \%)$ and $2.5 \%(2.5 \%)$, respectively for $\mathrm{Z} \rightarrow e^{+} e^{-}\left(\mathrm{Z} \rightarrow \mu^{+} \mu^{-}\right)$. A different way to search for any effect on $\mathrm{Z} \rightarrow l^{+} l^{-}$production due to the medium is to study the $\mathrm{Z}$ boson elliptic flow, $\mathrm{v}_{2}$ [12]. The $\mathrm{v}_{2}$ is defined as the amplitude of the second Fourier harmonic of the $\mathrm{Z}$ boson azimuthal emission angle with respect to the event plane [13]. The event plane contains the momentum vectors of the colliding nuclei, and is measured on an event-by-event basis from the azimuthal distribution of energy deposition in the FCal detectors.

\section{Results}

For both $\mathrm{Z} \rightarrow e^{+} e^{-}$and $\mathrm{Z} \rightarrow \mu^{+} \mu^{-}$analysis, the correction factors to account for the efficiency and detector resolution within the selected acceptance based on the simulation are calculated differentially 
in event centrality, $\mathrm{p}_{\mathrm{T}}$ and $\mathrm{y}^{\mathrm{Z}}$. In HI collisions, centrality reflects the overlap volume of the two colliding nuclei, controlled by the classical impact parameter. That is closely related to the average number of the participants $\left(<N_{\text {part }}>\right.$ ) and the number of binary collisions $\left(<N_{\text {coll }}>\right.$ ) between the nucleons of the colliding nuclei. The $\mathrm{Pb}+\mathrm{Pb}$ collision centrality is measured using the sum of transverse energy deposited in the FCal over the pseudorapidity range $3.2<|\eta|<4.9$ calibrated at the electromagnetic energy scale. In each decay channel, the correction factor is applied and the corrected background, estimated by the same-sign pairs, is subtracted. The two measurements are then statistically combined with weights set by their respective uncertainties. The totally corrected $\mathrm{Z}$ boson $\mathrm{p}_{\mathrm{T}}$ and $\mathrm{y}^{\mathrm{Z}}$ distributions are shown in Fig. 2. The data distributions plotted in $(0-80) \%$ centrality agree in shape with the PYTHIA simulations of $Z$ boson production in pp collisions.

The $\mathrm{Z}$ boson yields normalized by number of events and $\left\langle N_{\text {coll }}\right\rangle$, are shown in Fig. 3. The figure shows that the $\mathrm{Z} \rightarrow e^{+} e^{-}$and $\mathrm{Z} \rightarrow \mu^{+} \mu^{-}$scales with $<N_{\text {coll }}>$ within their uncertainties for all $\mathrm{p}_{\mathrm{T}}$ and centrality regions. A combined measurement error, including common uncertainties over the determination of $\left\langle N_{\text {coll }}>\right.$ is shown as the horizontal brackets. The dashed lines show constant fits to the combined data as a function of the average number $<N_{\text {part }}>$ of nucleons participating in the event.

The $\mathrm{v}_{2}$ of the $\mathrm{Z}$ boson in the $(0-60) \%$ centrality interval is measured to be $-0.015 \pm$ 0.018 (stat.) \pm 0.014 (sys.). This observation is an independent measurement consistent with $\mathrm{Z} \rightarrow l^{+} l^{-}$ yields being unaffected by the medium in HI collisions.

\section{Conclusions}

$\mathrm{Z}$ boson production has been measured in $\mathrm{Pb}+\mathrm{Pb}$ collisions at $\sqrt{s_{N N}}=2.76 \mathrm{TeV}$ using $0.15 \mathrm{nb}^{-1}$ of integrated luminosity collected in the $2011 \mathrm{LHC}$ run. Within $\left|\mathrm{y}^{\mathrm{Z}}\right|<2.5$, and $66 \mathrm{GeV}<\mathrm{m}_{l l}<102 \mathrm{GeV}$, a total of 772 and $1223 \mathrm{Z}$ boson candidates are reconstructed in the $\mathrm{Z} \rightarrow e^{+} e^{-}$and $\mathrm{Z} \rightarrow \mu^{+} \mu^{-}$channels respectively. The combinatorial background is at the level of $5 \%$ in the electron channel and $1 \%$ for the muon channel. $\mathrm{Z}$ boson yields integrated over $\left|\mathrm{y}^{\mathrm{Z}}\right|<2.5$ are consistent in the two channels in all measured $p_{\mathrm{T}}^{\mathrm{Z}}$ and centralities. The momentum and rapidity distributions of the $\mathrm{Z}$ bosons measured in $(0-80) \%$ centrality region are consistent in shape with the PYTHIA simulations of $Z$ boson production in pp collisions. Within the uncertainties the $\mathrm{Z}$ boson yield is found to be proportional to $\left\langle N_{\text {coll }}>\right.$. The elliptic flow coefficient $\mathrm{v}_{2}$ of the $\mathrm{Z}$ boson is consistent with zero within the uncertainties of the measurements.

\section{References}

[1] PHENIX Collaboration, K. Adcox et al., Nucl. Phys. A757 (2005) 184-283;

ATLAS Collaboration, G. Aad et al., Phys. Lett. B697 (2011) 294-312;

CMS Collaboration, S. Chatrchyan et al., Phys. Rev. Lett. 106 (2011) 212301.

[2] ATLAS Collaboration, ATLAS-CONF-2012-052, https://cdsweb.cern.ch/record/1451930.

[3] M. L. Miller et al., Ann. Rev. Nucl. Part. Sci. 57 (2007) 205243.

[4] ATLAS Collaboration, G. Aad et al., JINST 3 (2008) S08003.

[5] ATLAS Collaboration, G. Aad et al., Eur. Phys. J. C72 (2012) 1849.

[6] ATLAS Collaboration, G. Aad et al., Eur. Phys. J. C72 (2012) 1909.

[7] ATLAS Collaboration, G. Aad et al., Phys. Rev. D85 (2012) 072004.

[8] ATLAS Collaboration, G. Aad et al., Eur. Phys. J. C72 (2012) 2046.

[9] Sjostrand, S. Mrenna and P. Skands, JHEP 0605 (2006) 026. 

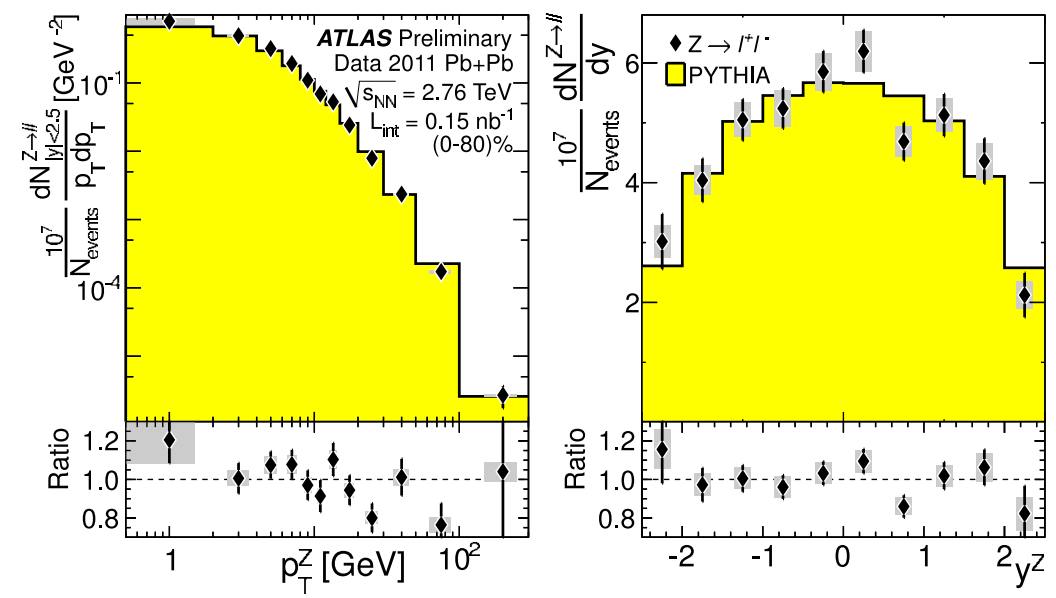

Figure 2. The $p_{\mathrm{T}}$ (left) and rapidity (right) distributions of $\mathrm{Z}$ bosons from lepton decay, compared to PYTHIA predictions normalized by area [2]. The error bars represent statistical uncertainties, and the filled bands represent systematic uncertainties. The lower panes display the ratio of data over PYTHIA.

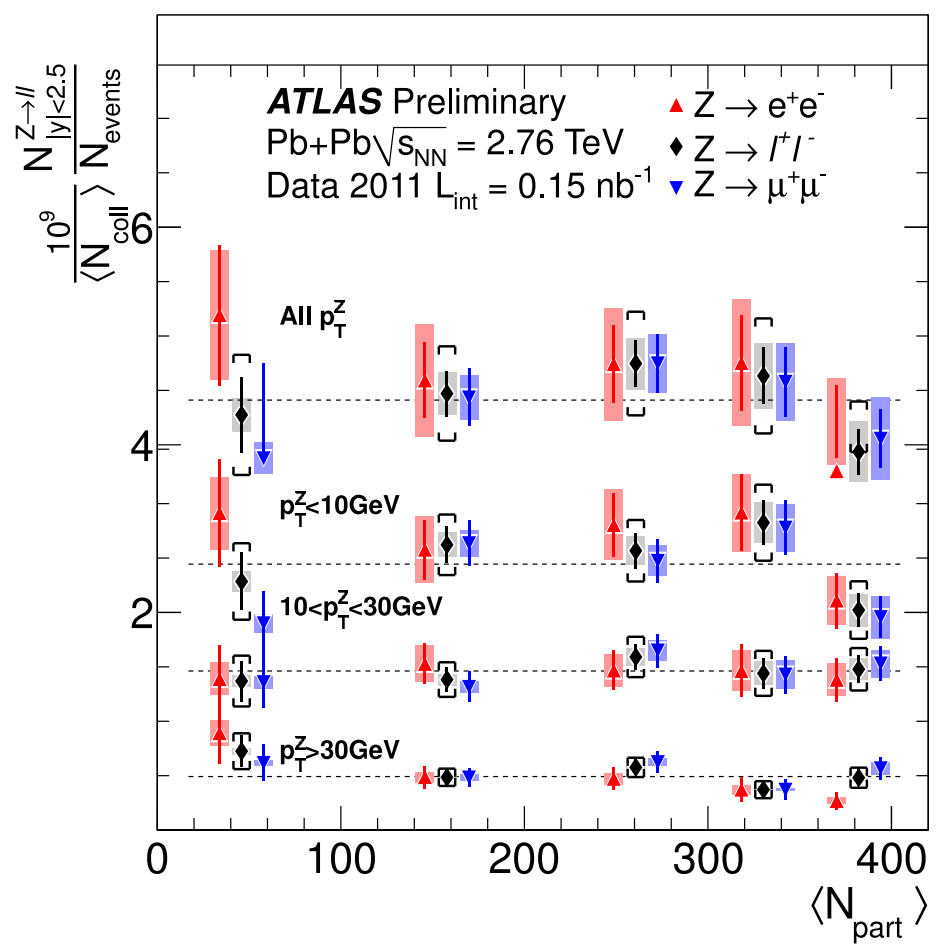

Figure 3. Centrality dependence of $Z$ boson yields divided by $<N_{\text {coll }}>$ in different intervals of $p_{\mathrm{T}}$ as a function of $<N_{\text {part }}>$ [2]. Results for $\mathrm{Z} \rightarrow e^{+} e^{-}$(upward pointing triangles) and $\mathrm{Z} \rightarrow \mu^{+} \mu^{-}$(downward pointing triangles) channels are shifted left and right respectively (for visibility) from their weighted average (diamonds) which 


\section{EPJ Web of Conferences}

[10] X.-N. Wang and M. Gyulassy, Phys. Rev. D44 (1991) 3501.

[11] S. Agostinelli et al., NIM A506 (2003) 250.

[12] A. Poskanzer and S. Voloshin, Phys. Rev. C58 (1998) 1671.

[13] ATLAS Collaboration, G. Aad et al., Phys.Rev. C86 (2012) 014907. 